
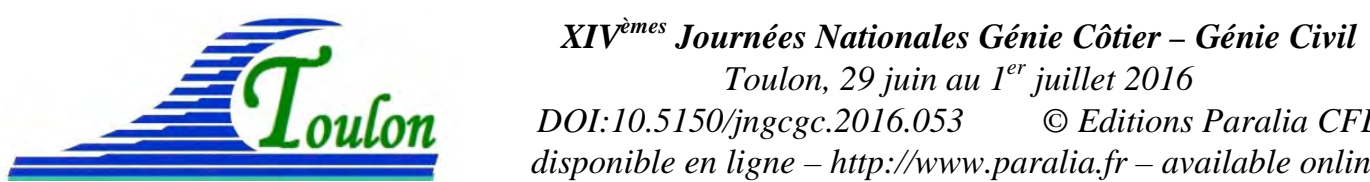

DOI:10.5150/jngcgc.2016.053 C Cditions Paralia CFL

disponible en ligne - http://www.paralia.fr - available online

\title{
SEM-REV, de 2007 à 2016, du projet au site opérationnel : retour d'expérience
}

\author{
Christian BERHAULT ${ }^{1}$, Izan LE CROM ${ }^{1}$, \\ Gérard LE BIHAN ${ }^{1}$, Jean-Marc ROUSSET ${ }^{1}$
}

1 Laboratoire de recherche en Hydrodynamique, Energétique et Environnement Atmosphérique, LHEEA, UMR 6598 CNRS - Ecole Centrale de Nantes1 rue de la Noë, BP 92101, 44321 Nantes, France.

jean-marc.rousset@ec-nantes.fr

\section{Résumé :}

Du projet envisagé en 2007 à l'accueil du premier prototype en mer en 2016, nous revenons sur la mise en place de SEM-REV au large du Croisic (44). Le développement du site dédié aux Energies Marines Renouvelables a été marqué par de nombreuses étapes. Les démarches administratives et les multiples aspects techniques de la construction sont relatés et les spécifications pour l'accueil d'un démonstrateur sur le site sont présentées. Un premier retour d'expériences issues des activités sur SEM-REV conclue l'article.

Mots-clés : Energies marines, Expérimentation, Essais en mer.

\section{Abstract:}

From the initial project in 2007 to the first prototype at sea in 2016, the different steps leading to the development of the field test site SEM-REV (Le Croisic, France) are described. A survey of the technical and administrative aspects is provided as well as some specifications for the installation of Marine Renewable Energy devices on site. A brief return of experience is presented, based on several on-going projects.

Keywords: Marine Energy, Experiments, Sea trials.

\section{La mise en place du projet SEM-REV}

Les travaux du LHEEA (ex-Laboratoire de Mécanique des Fluides) sur les énergies marines depuis plusieurs décennies ont conduit l'Ecole Centrale de Nantes à se lancer dans la construction d'un site d'essais pour tester les technologies des Energies Marines Renouvelables en milieu naturel. L'article revient sur la chronologie de la mise en place de ce Site En Mer pour la Récupération de l’Energie des Vagues et du Vent, SEM-REV, situé au large du Croisic en Loire-Atlantique.

\subsection{Le contexte règlementaire}

La mise en place du Site d'Essais en Mer pourrait être décrite comme un projet de recherche mais elle s'apparente tout de même à une course d'obstacle. Il s'est agi en 


\section{Thème 5 - Énergies et ressources marines}

effet d'obtenir un certain nombre d'autorisations des services de l'Etat et simultanément d'être en capacité d'obtenir les différents financements nécessaires à ces travaux, alors même que les cadres juridiques, techniques et financiers étaient imprécis.

En bref, la mise en place de SEM-REV repose sur des bases réglementaires pérennes concrétisées par une concession d'utilisation du Domaine Public Maritime (DPM) de longue durée (20 ans) pour tester des systèmes flottants ancrés, et par l'obtention de différentes autorisations pour, d'une part, réaliser les travaux d'installation des équipements et de raccordement sur le réseau et, d'autre part, pour opérer/exploiter le site d'essais en mer et à terre.

\subsection{Les grandes étapes de la mise en place de SEM-REV}

La construction du site d'essais s'est déroulée en 3 grandes étapes, entre 2007 et 2015, dont certains aspects ont fait l'objet de communications aux Journées Nationales Génie Côtier - Génie Civil (ROUSSET et al., 2010 et une lecture spéciale non publiée dans les actes du colloque de C. Berhault en 2014).

a) Les études préliminaires

La première étape de 2007 à 2010 a été constituée par plusieurs études préliminaires dont les résultats ont permis de guider le choix du site en mer sur des critères physiques, d'usages et d'impact environnemental, le choix du point de raccordement à terre (avec la localisation et les caractéristiques du poste de livraison, le passage du câble d'export), et le choix de l'installation du câble d'export en mer (sa route et son impact environnemental, les opérations marines à prévoir).

b) Les autorisations

La seconde étape est marquée par l'obtention de différentes autorisations entre 2010 et 2014. La Loi sur l'eau a conduit à mettre en place une concertation préalable avec les différents usagers (pêche, collectivités locales concernées, associations) et au lancement de plusieurs études d'impact environnemental. L'obtention de la concession d'utilisation du DPM a défini les conditions d'utilisation, du balisage et de signalisation du site pour assurer la sécurité maritime (suivant les recommandations de la Préfecture Maritime). A la suite de la première enquête publique, les autorisations pour les tests de houlomoteurs sont obtenues en 2011. La construction de la sous-station à terre et son raccordement électrique sont également autorisés.

En complétant le dossier de 2011 afin d'intégrer l'impact avifaune, l’impact paysager, les risques d'interférences radio-électriques et les contraintes de signalisation maritime et aérienne, l'autorisation pour tester des éoliennes flottantes est obtenue au début 2014.

c) La construction du site

Entre 2009 et 2015, de nombreux travaux permettent la mise en place des infrastructures du site aussi bien en mer qu’à terre. 


\section{XIV vèmes Journées Nationales Génie Côtier - Génie Civil \\ Toulon, 29 juin au $1^{\text {er }}$ juillet 2016}

En mer, on retiendra par exemple l'aménagement en mer, avec la mise en place du marquage de la zone et du déploiement de l'instrumentation scientifique (houle, courant, bouée météo), et l'installation du câble d'export électrique (câble statique de $20 \mathrm{kV}$ et 8MVA et doté de fibres optiques) qui a fait l'objet d'un forage dirigé sous la route puis sous l'estran complété d'un ensouillage jusqu'au centre du site en mer (figure 2).

En 2012, la réhabilitation dans le cadre du projet de locaux situés dans le parc de Pen Avel (parc du Conservatoire du Littoral géré par la Mairie du Croisic) a permis de doter l'ECN d'une base à terre pour accueillir l'équipe opérationnelle et les utilisateurs du site en mer. Par ailleurs la construction du poste de livraison pour le raccordement au réseau ERDF est issue d'une concertation menée avec les riverains vis-à-vis de l'impact paysager et du bruit généré en période de production (fonctionnement des transformateurs et ventilation des équipements).

Les raccordements sous-marins ont eu lieu à l'été 2015 avec la mise en place des éléments électriques (boîte de jonction, câble ombilical, connecteur) permettant de tester de 2 à 3 démonstrateurs simultanément et de les connecter au câble d'export.

Sur un plan opérationnel, au cours de ces étapes, l'ECN s'est adjoint une assistance à maîtrise d'ouvrage et un maître d'œuvre qui a notamment assuré le suivi des principaux lots attribués dans le cadre des marchés publics: les équipements électriques (dont le câble) et les travaux d'installation (terrestre et maritime).

Des travaux complémentaires sont en cours d'achèvement avec l'installation ce printemps 2016 d'une liaison en fibre optique entre la base de Pen Avel, le poste de livraison et le nouveau réseau haut débit de la Presqu'île de Guérande. Ces nouvelles capacités de communications vont faciliter les échanges numériques sécurisés avec le campus de l'ECN à Nantes (moyens informatiques du laboratoire, centre de calcul, etc.) et avec les partenaires des démonstrateurs pour la supervision à distance des équipements déployés sur le site d’essais (éolienne, houlomoteur, ...).

\subsection{Bilan de la construction}

Le site d'essais en mer SEM-REV (figure 1) est considéré comme pleinement opérationnel depuis l'automne 2015 et prêt à accueillir un prototype dans les conditions de raccordement au réseau électrique (LE CROM, 2016).

Il est évident que la définition puis la mise en place de tous les équipements, la validation de toutes les procédures etc., ont induit de nombreux décalages sur le planning initial. A l'issue des études préliminaires, il aura fallu près de 5 ans d'efforts continus pour être en capacité d'accueillir un premier démonstrateur.

\subsection{Les aspects financiers}

La complexité financière du projet, avec un budget final des investissements proche de 15M€ (soit pratiquement 19M€ TTC), a été un défi par lui-même. Il a pu être relevé par 


\section{Thème 5 - Énergies et ressources marines}

sa prise en compte tout d'abord dans le Contrat de Projets de l'Etat en Région Pays de la Loire (CPER 2007 - 2013) puis, plus récemment, dans le Programme des Investissements d'Avenir (PIA) en particulier pour le raccordement sous-marin de 2015. Il est à noter que l'enveloppe financière en 2007 avait été estimée à 5.8M€. A cette époque, le seul centre d'essais en mer existant est l'EMEC situé aux Orcades (Ecosse) et son fonctionnement n'est alors pas encore totalement pérennisé. La communauté scientifique des EMR manque donc de recul sur les difficultés à venir et tend peut être à sous-estimer les délais administratifs et réglementaires ainsi que les coûts engendrés par les éléments technologiques à mettre en place pour constituer les infrastructures d'un centre d'essais (le câble, le raccordement, etc.).

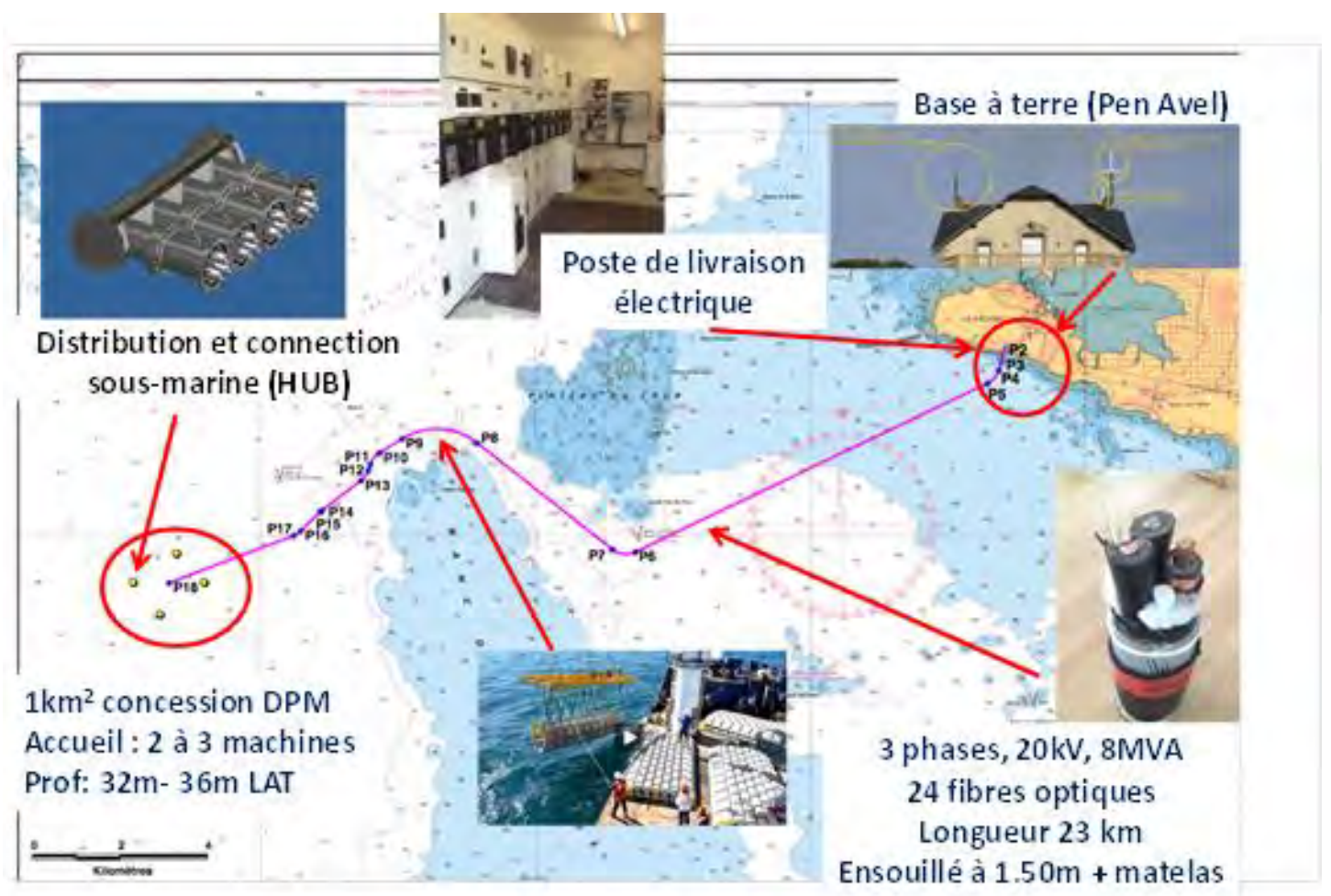

Figure 1. Les équipements électriques de SEM-REV.

L'expérience va montrer que tous ces éléments, nommés maintenant briques technologiques, sont aussi des prototypes et que les opérations marines pour les mettre en place sont également inédites. Des conditions extérieures perturbent également les estimations financières, comme le cours du cuivre (en forte hausse à l'époque) constituant les câbles ou encore les taux d'affrètement des navires variant avec le coût des énergies fossiles.

Les budgets associés sont donc revus à la hausse au cours du temps en prenant en compte également les besoins croissants de la filière EMR qui est en plein développement (plus de machines à tester, plus grande puissance, ...). 


\section{XIV ${ }^{\text {èmes }}$ Journées Nationales Génie Côtier - Génie Civil \\ Toulon, 29 juin au $1^{\text {er }}$ juillet 2016}

Les règles budgétaires, notamment européennes, vont également obliger à revoir les plans financiers initiaux. Pour rester une structure de recherche, SEM-REV ne doit en effet bénéficier que de fonds publics au travers de différents supports et accompagnements et de projets de recherche.

Au final, le financeur principal de SEM-REV est la Région des Pays de la Loire et les financements complémentaires, outre ceux de l'Etat, viennent du Département de Loire Atlantique, du fond FEDER (Fonds Européens de Développement Régional) et des contributions du CNRS et de l'ECN.

Depuis 2015 et pour une durée de 6 ans, le PIA poursuit son soutien à SEM-REV par le co-financement, via l'Agence Nationale de la Recherche, des coûts de fonctionnement du site d'essais lorsque ces dépenses sont générées par les projets de recherche accueillis sur la zone en mer.

Actuellement une dernière phase administrative est en cours d'achèvement avec la contractualisation du raccordement sur le réseau ERDF et la contractualisation du rachat de l'électricité produite par les machines testées en mer.

\section{La mise en place des essais}

L'accueil d'un démonstrateur sur le site d'essais en mer s'effectue sur la base d'un cahier des charges qui traite des aspects techniques, règlementaires, juridiques et financiers. Le tableau suivant reprend les principales spécifications à prendre en compte pour le bon déroulement de la procédure.

Tableau 1. Eléments de la procédure pour l'accueil d'un démonstrateur en mer.

\begin{tabular}{|c|c|}
\hline Rubrique & Exemples des éléments à prendre en compte \\
\hline L'environnement du site & $\begin{array}{l}\text { conditions océano-météo, la nature du sol, profondeur d'eau, emprise } \\
\text { possible (flotteur et ancrage), état environnemental initial }\end{array}$ \\
\hline $\begin{array}{l}\text { Les équipements électriques } \\
\text { existants et les contraintes } \\
\text { associées }\end{array}$ & $\begin{array}{l}\text { qualité du signal produit, contraintes ERDF, équipements nécessaires } \\
\text { à bord dont ceux pour la communication, le traitement des alarmes, } \\
\text { les redondances,... }\end{array}$ \\
\hline Le démonstrateur & $\begin{array}{l}\text { architecture, ancrage, raccordement, système de production (Power- } \\
\text { Take-Off) et équipements électriques de transformation et de } \\
\text { protection }\end{array}$ \\
\hline La sécurité maritime & $\begin{array}{l}\text { risques et prévention, surveillance et intervention, assurance, } \\
\text { interaction entre démonstrateurs, procédures d'intervention sur site }\end{array}$ \\
\hline Le suivi environnemental & $\begin{array}{l}\text { répercussion des conditions imposées dans les annexes des arrêtés } \\
\text { préfectoraux et dans la concession d'utilisation du DPM }\end{array}$ \\
\hline Les conditions contractuelles & $\begin{array}{l}\text { la planification des essais, l'estimation des coûts, la provision pour } \\
\text { démantèlement, la disponibilité }\end{array}$ \\
\hline
\end{tabular}




\section{Thème 5 - Énergies et ressources marines}

A l'issue de ces discussions préalables, le processus de préparation des essais est enclenché reposant sur les étapes chronologiques suivantes :

i. La signature d'un accord de confidentialité, préalable au montage du projet, permettant l'échange des informations entre l'ECN et le développeur

ii. Une étude de faisabilité de l'implantation du démonstrateur sur SEM-REV : ressource, environnement, adaptation, raccordement électrique, planning, budget et financement, accord de consortium..

iii. Le montage du projet, la réservation d'un emplacement, le planning et le cadre budgétaire. Le projet peut être mené dans le cadre européen ou national mais il est imposé par la réglementation européenne que le démonstrateur soit testé dans le cadre d'un projet collaboratif dont l'ECN doit être partenaire et en charge de la partie du projet concernant l'utilisation de SEM-REV.

iv. La signature du contrat de projet et la finalisation des annexes techniques au contrat, intégrant les évolutions du démonstrateur pour son adaptation au site qui ne seront pas en mesure d'être définies à la signature du contrat.

v. La soumission d'un dossier de présentation du démonstrateur et du programme d'essais aux autorités administratives afin de valider le respect des conditions associées aux Autorisations et la règlementation en vigueur. Le dossier doit également présenter les opérations d'installation et de démantèlement sur site, la stratégie de surveillance et de maintenance, les plans de prévention.

vi. La mise en place du planning des essais. Cette étape peut être difficile car il est nécessaire de prévoir les éventuelles périodes d’arrêt, voire de retrait, du prototype au cours des essais, les pannes, les périodes de maintenances électriques, les interactions avec les autre machines déployées sur le site, ...

Il apparaît donc que ces essais en mer ne peuvent être mis en place sans une étroite et préalable collaboration entre le développeur et l'ECN. Une configuration analogue est suivie par les autres sites européens d'essais en mer, une harmonisation des procédures d’accueil étant par ailleurs en cours.

\section{Le contexte scientifique des essais en mer}

Sous la responsabilité de l'ECN et soutenues financièrement par le PIA (cf 1.4), les activités de SEM-REV sont intégrées dans un programme de Recherche \& Développement qui s'articule autour de 4 axes majeurs intégrant des projets collaboratifs eux-mêmes impliquant partenaires académiques et industriels :

- Axe 1: Les paramètres de l'environnement marin, pour la ressource, le dimensionnement et l'impact environnemental des EMR

- Axe 2 : Les technologies de production EMRs et de stockage : éoliennes flottantes, houlomoteurs et technologies hybrides, ...

- Axe 3 : La conversion, le transport et le stockage de l'énergie 


\section{XIV èmes Journées Nationales Génie Côtier - Génie Civil \\ Toulon, 29 juin au $1^{\text {er }}$ juillet 2016}

- Axe 4: La surveillance, la sécurité, la maintenance et les opérations en mer, la formation

Une illustration de projets en cours dans les axes 1 et 2 est succinctement présentée, l'une concernant les activités scientifiques pour le suivi environnemental et l'autre un démonstrateur en mer.

\subsection{Le suivi des organismes benthiques}

Dans le cadre des différentes autorisations délivrées par l'Etat (annexes des arrêtés préfectoraux et concession d'utilisation du DPM), il est demandé un suivi des organismes benthiques le long du câble (d'export électrique) statique et sur la zone en mer.

A cette fin, des prélèvements d'organismes benthiques sont effectués deux fois par an. La figure 2 présente les positions des 10 stations sélectionnées pour le suivi, réparties dans différents faciès sédimentaires et déterminés à la suite de campagnes effectuées entre 2008 et 2010 pour les études préliminaires ( $c f$. la partie 1.2 de l'article).

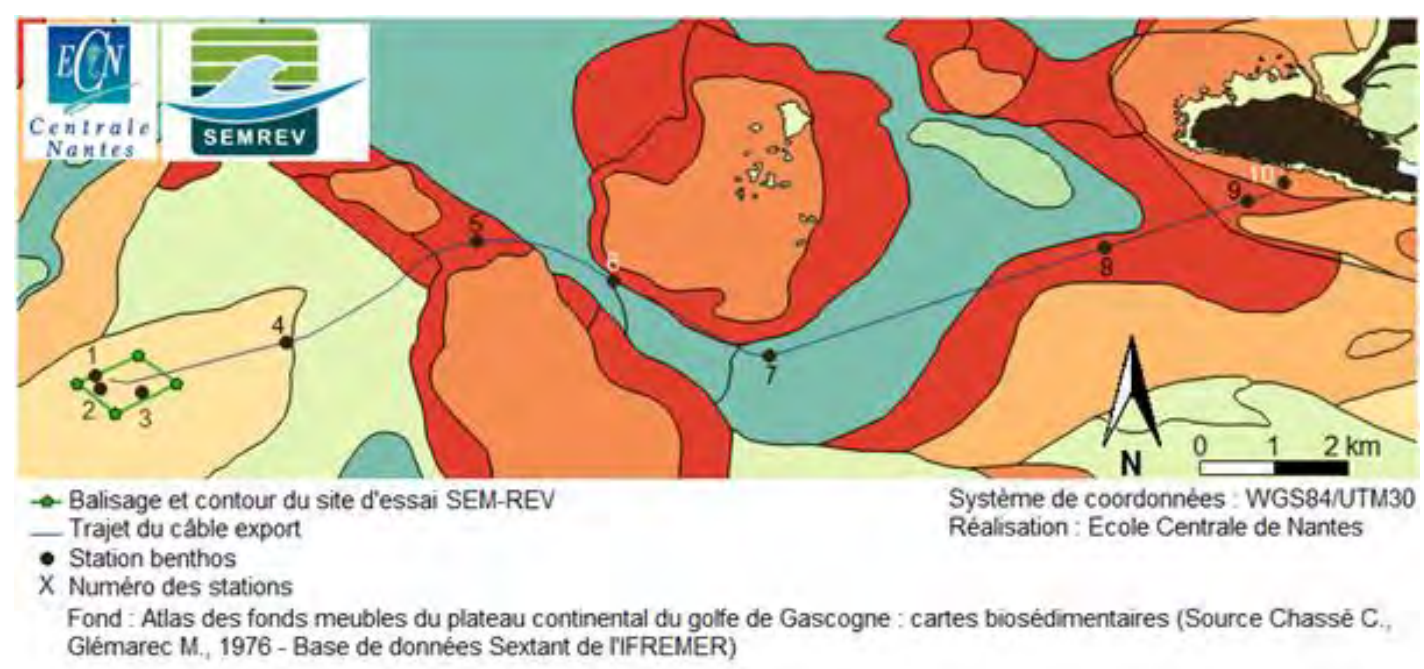

Figure 2. Les positions des stations de suivi des organismes benthiques.

La première campagne de suivi benthique pour SEM-REV a été effectuée en octobre 2015. Elle a été entamée après l'achèvement des travaux de raccordement au câble statique. Ses résultats constituent en quelque sorte un état initial du site avant l'arrivée de démonstrateurs. Une seconde campagne de prélèvements a été réalisée en mars 2016, à la sortie des conditions hivernales. L'analyse des résultats est en cours au moment de la rédaction du manuscrit.

Le suivi sur une durée longue (sur 2 ou 3 ans) devra permettre de mettre en évidence des variations éventuelles liées aux activités EMR du site. Toutefois des difficultés d'analyse sont attendues car les zones de mesures sont également tributaires d'une importante variabilité saisonnière et des interactions avec l'estuaire de la Loire. 


\section{Thème 5 - Énergies et ressources marines}

\subsection{La mesure des conditions environnementales}

L’instrumentation déployée sur SEM-REV est opérationnelle depuis 2009. Elle est principalement constituée de bouées houlographes, de profileurs courantométriques (ADCP) et d'une bouée météo.

Le maintien sur zone de ces éléments est un défi permanent en particulier vis-à-vis de l'intégrité des ancrages et de la sécurité maritime. La figure 3 montre le trajet d'une bouée houlographe dans les premiers jours de janvier 2016 après la rupture de sa ligne de mouillage et avant sa récupération en mer. La trajectoire issue des relevés GPS est complexe et illustre la difficulté de prédire les déplacements au cours du temps de ce type d'objet (épave, conteneur etc.). Seule la transmission de sa position à la base à terre permet de la retrouver puis de la mouiller sur le site de nouveau.

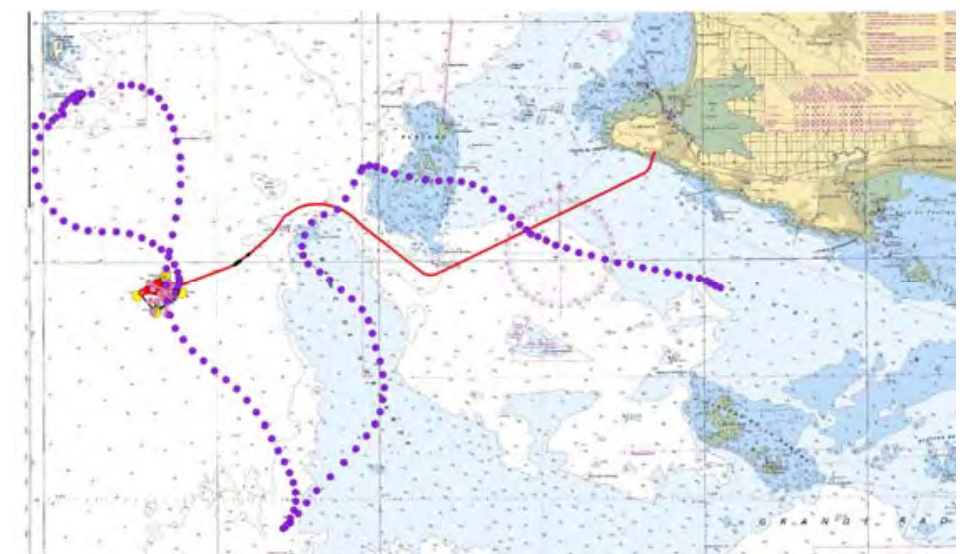

Figure 3. Suivi des positions GPS d'un houlographe en dérive (janvier 2016).

\subsection{La bio-colonisation}

Outre les conditions de houle qui peuvent être sévères en hiver, les équipements déployés sur le site doivent faire face également au développement rapide de la biocolonisation. Les photos de la figure 4 illustrent les modifications géométriques induites par la présence des moules sur un houlographe (à la sortie de l'eau au centre et après nettoyage à gauche) et sur les lignes d'ancrage.

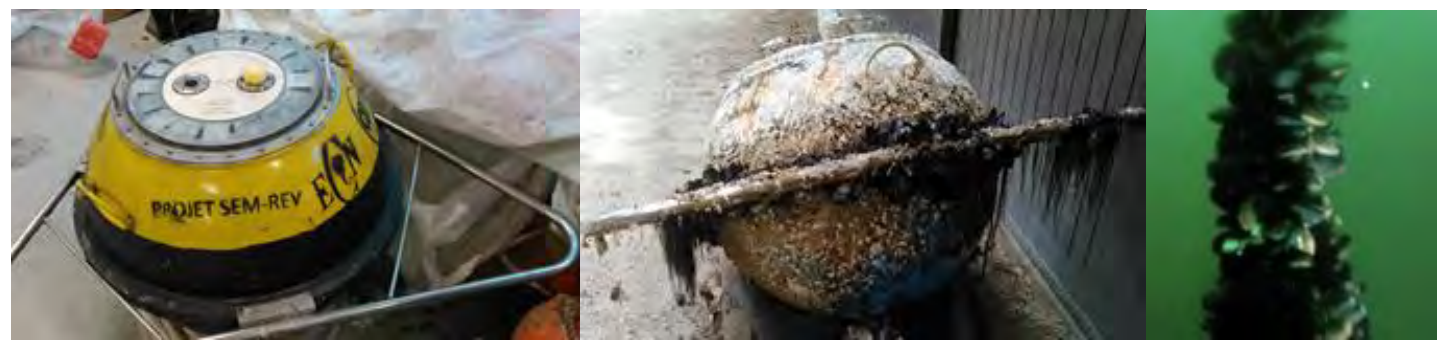

Figure 4. Photographies montrant la biocolonisation sur une bouée de mesure de houle et sur une ligne d'ancrage de la bouée météo (à droite). 


\section{XIV èmes Journées Nationales Génie Côtier - Génie Civil \\ Toulon, 29 juin au $1^{\text {er }}$ juillet 2016}

La biocolonisation entraîne par exemple l'augmentation du diamètre des lignes et elle contribue alors à un fort accroissement des efforts de traînée induits par les écoulements (houle, courant) sur les ancrages. Les liaisons avec la bouée en surface sont ainsi plus sollicitées et les structures fatiguent plus rapidement que les estimations, ce qui peut conduire à une rupture de ligne d'ancrage.

Cette biocolonisation contribue également à diminuer la flottabilité des houlographes qui tendent alors à rester entièrement submergés (au lieu de rester en surface) et ne pas mesurer correctement les hauteurs des vagues.

La rapidité de la biocolonisation sur les équipements et les risques de dégradations imposent donc de fréquents contrôles sur le site et des nettoyages réguliers (environ tous les 2 mois).

\subsection{Le projet FP7-Floatgen}

Floatgen est un projet européen (FP7) de démonstrateur d'éolien flottant. Ce projet devrait permettre de confirmer la performance de la combinaison d'une éolienne (une turbine de 2MW) et de la fondation flottante conçue par Ideol (figure 5). L'installation du démonstrateur sur SEM-REV est planifiée pour durer 2 ans à partir de fin 2016.

L'instrumentation déployée sur le site (mesure de la houle, des courants et du vent) ainsi que celle embarquée spécifiquement sur le démonstrateur (tensions dans les lignes d'ancrage, mouvements de la fondation flottante, etc.) permettront de valider les travaux effectués en bassin d'essais et en simulation numérique et de constituer ainsi une exceptionnelle base de données expérimentales.

Le raccordement électrique au réseau ERDF permettra de confronter aux mesures effectives, les hypothèses sur la capacité de production énergétique du prototype. Ces données contribueront par ailleurs à vérifier les schémas de développement des futurs parcs d'éoliennes flottantes.

La figure 4 (à gauche) donne une vue de l'implantation des différentes lignes d'ancrage et des câbles électriques qui seront présents sur le site en 2016 et 2017. Il apparaît que les opérations marines pour la maintenance et l'installation seront compliquées par la présence de tous ces "obstacles".

Les interventions sur les démonstrateurs devront donc être bien planifiées temporellement mais surtout spatialement sous peine d'accrocher et d'endommager les équipements relativement fragiles par des navires déployés sur la zone. En cela ces travaux de planification pour les opérations sur SEM-REV préfigurent les difficultés d’installation et de maintenance des parcs de houlomoteurs et d'éoliennes. 


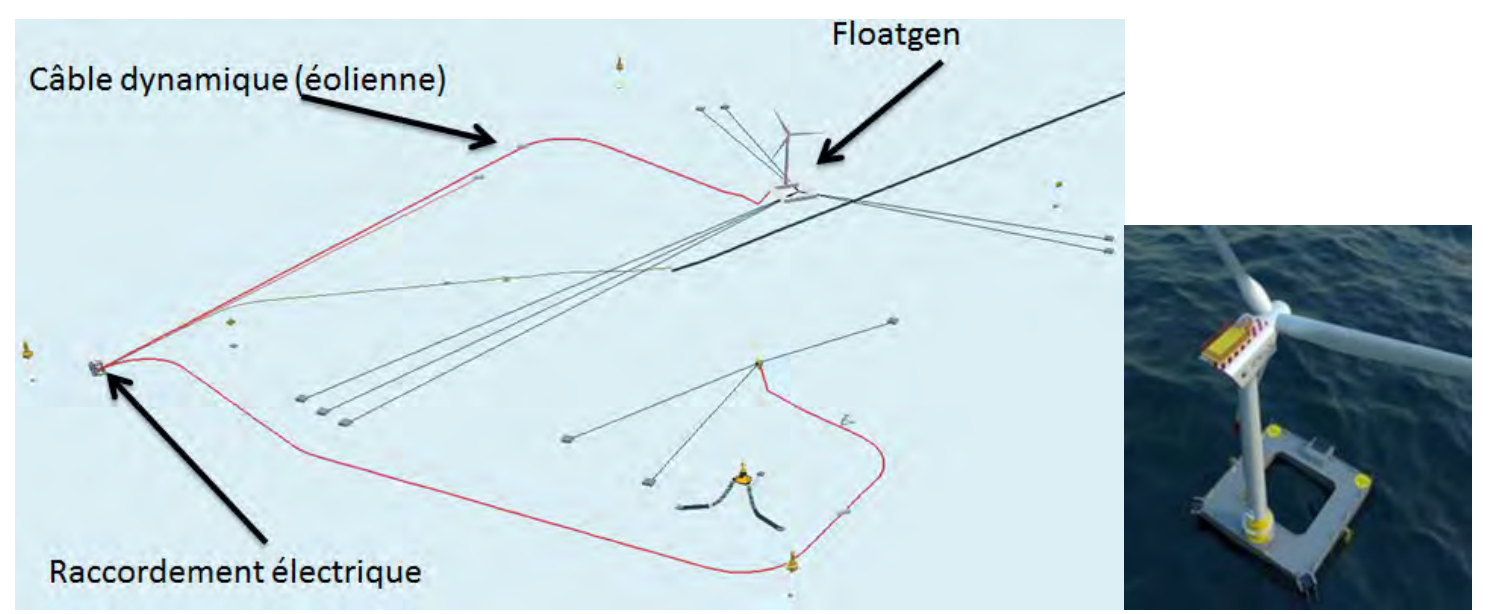

Figure 5. Dispositions des lignes électriques et d'ancrages (à gauche) et impression du démonstrateur Floatgen (à droite).

\section{Conclusion}

Après ces années consacrées à un long travail de mise en place, SEM-REV entre maintenant dans une phase opérationnelle enthousiasmante qui va apporter également son lot de surprises. Les difficultés seront toutefois certainement plus techniques et environnementales qu'administratives, et le site va pouvoir accueillir des projets ambitieux, tels qu'une éolienne flottante (projet FP7 Floatgen porté par Ideol) et un dispositif houlomoteur (projet porté par Geps-Techno). SEM-REV est donc à même de contribuer pleinement à la validation des technologies des Energies Marines Renouvelables.

\section{Remerciements :}

Les remerciements vont aux membres de l'équipe SEM-REV qui contribuent au fonctionnement du site ainsi qu'à tous les personnels administratifs et scientifiques de l'ECN et du LHEEA impliqués dans cette aventure depuis de nombreuses années.

Enfin les remerciements vont aux soutiens financiers du projet : le Conseil Régional des Pays de la Loire, l'Etat, le Conseil départemental de Loire-Atlantique, le Centre National de la Recherche Scientifique (CNRS) et l'Agence Nationale pour la Recherche.

\section{Références}

LE CROM I. (2016). Feedback on the implementation of the SEM-REV test site for Marine Renewable Energy. International Conference on Ocean Energy, Edinburgh. ROUSSET J-M, MOUSLIM H, LE BIHAN G., BABARIT A. (2010). Le projet SEM$R E V$ : un site d'expérimentation en mer pour la recherche et l'industrie. Journées Génie Civil - Génie Côtier, Sables d’Olonne, pp 813-822. http://dx.doi.org/10.5150/jngcgc.2010.090-R 